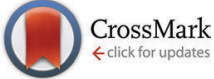

Cite this: Phys. Chem. Chem. Phys., 2015, 17, 31357

\author{
Luca Frediani ${ }^{\star a}$ and Dage Sundholm ${ }^{\star b}$
}

\title{
Real-space numerical grid methods in quantum chemistry
}

DOI: $10.1039 / c 5 c p 90198 g$

www.rsc.org/pccp

Real-space numerical electronic structure methods have lately attracted increasing scientific attention as they are mathematically robust, accurate and well suited for modern, massively parallel computing resources. Despite these significant advantages, numerical quantum chemistry methods are not yet the mainstream approach because they are computationally expensive and can have prohibitive memory requirements. In addition, the scientific community, to some extent, lacks widely available software with standard features to perform routine calculations of molecular properties, such as structures, excited states, solvent effects, electric and magnetic (hyper)polarizabilities, etc. The present Real-Space Numerical Grid Methods in Quantum Chemistry issue of Physical Chemistry Chemical Physics (PCCP) is an attempt to support the development of numerical quantum chemistry methods by gathering articles in a variety of research areas, such as numerical mathematics, computer science, quantum physics, quantum chemistry, and materials science. Thus, the articles in the issue cover a wide spectrum of the scientific expertise of the research field. A further aim of the themed issue is to draw attention to this challenging

\footnotetext{
${ }^{a}$ Centre for Theoretical and Computational Chemistry, Department of Chemistry, UiT The Arctic University of Norway, N-9037 Tromsø, Norway. E-mail: luca.frediani@uit.no

${ }^{b}$ Department of Chemistry, University of Helsinki, A. I. Virtanens Plats 1, P. O. Box 55, FI-00014, Finland. E-mail:Dage.Sundholm@helsinki.fi
}

and very promising research area. The contributions to this special issue show that we are approaching a turning point: from a niche of proof-of-principle calculations, local basis functions are becoming a viable alternative in a growing spectrum of applications.

The first numerical electronic structure methods for molecular calculations were developed more than 30 years ago. ${ }^{1-8}$ The early implementations showed that a very high accuracy can indeed be obtained. However, large scale applications on general molecules were hampered due to the huge computational costs. Since the development of the pioneering numerical electronic structure methods, a large number of numerical electronic structure approaches and codes have emerged. ${ }^{9-26} \mathrm{~A}$ significant boost to the field has come with the evolution of modern, massively-parallel computer architectures: in the last few years the increase in computational power has mostly been due to the advance of large clusters with lots of processors, rather than the increased performance of the individual CPUs, the speed of which, by widespread consensus, is flattening out due to the limits in physical laws. Traditional computational methods face significant challenges to exploit the full potential of such clusters, whereas numerical approaches with local bases on real-space grids are better suited to distributing huge numbers of relatively simple computational operations on a large number of CPUs. Additional speedup can also be obtained by employing GeneralPurpose Graphics Processing Units (GPGPUs).

The present PCCP issue gathers contributions from many of the leading method developers in this field. The themed issue contains 21 original articles covering a broad variety of aspects. The optimization and construction of grids, the use of wavelet methods in quantum chemistry studies, real-space implementations in Density Functional Theory (DFT) programs, tensorial and data compression techniques, and numerical calculations of excited states including ab initio correlation approaches are among the research topics which are covered.

The multiwavelet methods presented by Yanai et al. (DOI: 10.1039/C4CP05821F) and Kottmann et al. (DOI: 10.1039/ $\mathrm{C} 5 \mathrm{CP} 00345 \mathrm{H}$ ) demonstrate the feasibility of solving the time-dependent DFT, linear-response Hartree-Fock, and the related Configuration Interaction Singles (CIS) equations numerically yielding results for excited states. Kim et al. (DOI: 10.1039/C5CP00352K) used a more traditional excited-state approach by expanding the molecular orbitals in Lagrange-sinc functions and considering the occupied molecular orbitals and only lowest-lying virtual Hartree-Fock or DFT orbitals in the CIS calculation.

The grid size determines the accuracy and memory requirements. Grid-optimizations, quadratures and algorithms that reduce the number of grid points (local basis 
functions) are therefore of central importance. For example, refining methods including error control is the key to the success of the wavelet-based approaches. The main disadvantage of the refinement is the significant increase of parameters at the finest level. Several contributions address this question: Nagy and Pipek (DOI: 10.1039/C5CP01214G) have developed a wavelet algorithm that can be used for predicting the resolution coefficients at the finer levels in an efficient way. Bugeanu et al. (DOI: 10.1039/C5CP03410H) use wavelets to consider solvent effects within the polarizable continuum model. The adaptive finite element method discussed by Tsuchida et al. (DOI: 10.1039/C5CP00320B) is another approach to reduce the grid size or to increase the accuracy for a given number of grid points. Zuzovski et al. (DOI: 10.1039/ C5CP01090J) employ auxiliary grids to increase the accuracy and computational efficiency. Genovese and Deutsch (DOI: 10.1039/C5CP01236H) discuss multipolepreserving quadratures in their article, whereas Baye and Dohet-Eraly (DOI: 10.1039/C5CP00110B) use Lagrange meshes to increase the accuracy when the quantum systems are exposed to strong confinement effects. In the short review article, Andrade et al. (DOI: 10.1039/C5CP00351B) discuss the main features and methods of the Octopus code that employs numerical basis functions. Tensorial methods and data compression are alternative methods for reducing the memory requirement and improving the computational accuracy. Khoromskaia and Khoromskij (DOI: 10. 1039/C5CP01215E) present a review of tensor decomposition techniques in the context of electronic structure calculations on molecules using local basis functions. Flad et al. (DOI: 10.1039/C5CP01183C) make use of singular analysis - an abstract mathematical framework - to construct useful interaction operators and to understand the origin of the shape of the electronic wave function close to the nuclei as well as at short interelectronic distances. Another strategy to obtain high accuracy and efficiency for long-ranged interelectronic interactions is constituted by multipole expansions: linear scaling is eventually achieved when the electrostatic moments of different domains are grouped together before combining them to the corresponding interaction energy contribution. Toivanen et al. (DOI: 10.1039/ C5CP01173F) have developed and implemented a grid-based fast multipole moment Fast Multiple Method (FMM) scheme to calculate two-electron energies: the multipole moments are calculated numerically for subdomains and combined to larger domains when feasible. They show that the FMM calculations approach the $N(0)$ efficiency, i.e. the computational time is independent of the system size (Amdahl's law) when enough GPGPUs are available.

Eight of the articles are devoted to numerical DFT methods, codes and algorithms. Hu et al. (DOI: 10.1039/ C5CP00333D) have employed their Discontinuous Galerkin Density Functional Theory (DGDFT) methodology in studies on carbon nanoribbons. In the massively parallel $a b$ initio Molecular Dynamics (AIMD) calculations used to study thermodynamic stability, they found that the DGDFT approach scales well up to 50000 processors. Beck (DOI: 10.1039/ $\mathrm{C} 5 \mathrm{CP} 01222 \mathrm{H})$ presents in his article a novel alternative stochastic method that solves directly the one-electron density matrix in real space. The idea and the underlying theory as well as the first implementation for simple model systems are presented. One important aim of his article is to stimulate further efforts in the development of methods which build upon the stochastic sampling ideas. Chakraborty et al. (DOI: 10.1039/ C5CP00995B) have developed an orbital free DFT approach, whose quantum hydrodynamic equations are solved numerically using an implicit Euler-type real space method. Nakata et al. (DOI: 10. 1039/C5CP00934K), as well as Mohr et al. (DOI: 10.1039/C5CP00437C), discuss two linear-scaling DFT approaches with localized support functions that can be applied on very large molecular systems when employing massively parallel computers. Espinosa Leal et al. (DOI: 10.1039/ C5CP01211B) discuss the implementation and application of an orbital-free DFT approach that employs a numerical basis set. They prefer such an approach because numerical difficulties often arise when other kinds of basis sets are employed. Bobbitt et al. (DOI: 10.1039/C5CP02561C) have developed an accurate integration technique that is used in molecular structure optimizations and in calculations of second derivatives of the potential energy surface in real space calculations. Natan (DOI: 10.1039/C5CP01093D) describes a three-dimensional (3D) real-space implementation of the Fock exchange using the Krieger-Li-Iafrate (KLI) approximation in the Optimized Effective Potential (OEP) approach.

We believe that the articles in this themed issue show how real-space numerical methods for electronic structure calculations will further develop, from a niche for specialists to being one of the most common approaches of quantum chemistry and molecular physics. The current mainstream approaches have evolved from a past where the limits in computational power imposed drastic choices on the basis functions (atomic orbitals for molecules and periodic functions for crystals) in order to minimize the computational effort. The exponential increase of computational power, and the deployment of ever larger distributed architectures open the way for methods and algorithms that are well suited to harvest these resources, such as those presented in this themed issue.

The use of numerical methods makes it possible to reduce the basis set truncation error far below chemical accuracy. Gauge-origin problems appearing when performing calculations that consider interactions with external magnetic fields will disappear, because the employed numerical basis sets are practically complete (to within any given threshold). It will be possible to implement different types of density functionals because of the local properties of the basis functions. Schrödinger equations with terms considering explicit correlation effects have been tackled using numerical methods. However, we don't want to underestimate the challenges that real-space numerical methods face: one fundamental problem is constituted by electron correlation effects beyond DFT or ab initio SelfConsistent Field (SCF) approaches, which is still an open question for such methods because of the so called "curse of dimensionality"; ${ }^{21}$ one technical issue, strictly connected to the electron correlation problem, is the large memory requirements which are currently limiting the 
applicability of real-space methods to a larger scale. Other important issues are the calculation of higher order molecular properties and the inclusion of time development. There is still much work to be done.

\section{Acknowledgements}

The Graphical Abstract image includes images reproduced from four papers contained in this issue.

\section{References}

1 E. A. McCullough Jr., Comput. Phys. Rep., 1986, 4, 267-312.

2 D. Becke, J. Chem. Phys., 1982, 76, 6037-6045.

3 L. Laaksonen, P. Pyykkö and D. Sundholm, Comput. Phys. Rep., 1986, 4, 315-344.

4 W. Schulze and D. Kolb, Chem. Phys. Lett., 1985, 122, 271-275.

5 D. Heinemann, D. Kolb and B. Fricke, Chem. Phys. Lett., 1987, 137, 180-182.

6 D. Sundholm, J. Olsen, P. A. Malmqvist and B. O. Roos, Numerical Determination of the Electronic Structure of Atoms, Diatomic and Polyatomic Molecules, Proc. NATO Advanced Research
Workshop, Versailles, M. Defranceschi and J. Delhalle (ed.), Reidel, Dordrecht, 1989, pp. 329-334.

7 A. D. Becke and R. M. Dickson, J. Chem. Phys., 1990, 92, 3610-3612.

8 J. Kobus, L. Laaksonen and D. Sundholm, Comput. Phys. Commun., 1996, 98, 346-358.

9 J. R. Chelikowsky, N. Troullier, K. Wu and Y. Saad, Phys. Rev. B: Condens. Matter Mater. Phys., 1994, 50, 11355-11364.

10 J. M. Soler, E. Artacho, J. D. Gale, A. García, J. Junquera, P. Ordejón and D. Sánchez-Portal, J. Phys.: Condens. Matter, 2002, 14, 2745-2779.

11 R. J. Harrison, G. I. Fann, T. Yanai, Z. T. Gan and G. Beylkin, J. Chem. Phys., 2004, 121, 11587-11598.

12 T. Yanai, G. I. Fann, Z. T. Gan, R. J. Harrison and G. Beylkin, J. Chem. Phys., 2004, 121, 2866-2876.

13 T. Yanai, G. I. Fann, Z. T. Gan, R. J. Harrison and G. Beylkin, J. Chem. Phys., 2004, 121, 6680-6688.

14 D. Sundholm, J. Chem. Phys., 2005, 122, 194107.

15 J. J. Mortensen, L. B. Hansen and K. W. Jacobsen, Phys. Rev. B: Condens. Matter Mater. Phys., 2005, 71, 035109.
16 D. R. Bowler, R. Choudhury, M. J. Gillan and T. Miyazaki, Phys. Status Solidi B, 2006, 243, 989-1000.

17 A. Castro, H. Appel, M. Oliveira, C. A. Rozzi, X. Andrade, F. Lorenzen, M. A. L. Marques, E. K. U. Gross and A. Rubio, Phys. Status Solidi B, 2006, 243, 2465-4883.

18 T. Shiozaki and S. Hirata, Phys. Rev. A: At., Mol., Opt. Phys., 2007, 76, 040503.

19 J. D. Talman, Phys. Rev. A: At., Mol., Opt. Phys., 2010, 82, 052518.

20 F. A. Bischoff and E. F. Valeev, J. Chem. Phys., 2011, 134, 104104.

21 F. A. Bischoff, R. J. Harrison and E. F. Valeev, J. Chem. Phys., 2012, 137, 104103.

22 S. A. Losilla and D. Sundholm, J. Chem. Phys., 2012, 136, 214104.

23 G. Schofield, J. R. Chelikowsky and Y. Saad, Comput. Phys. Commun., 2012, 183, 497-505.

24 L. Frediani, E. Fossgaard, T. Flå and K. Ruud, Mol. Phys., 2013, 111, 1143-1160.

25 S. Mohr, L. E. Ratcliff, P. Boulanger, L. Genovese, D. Caliste, T. Deutsch and S. Goedecker, J. Chem. Phys., 2014, 140, 204110.

26 S. Losilla, M. A. Watson, A. AspuruGuzik and D. Sundholm, J. Chem. Theory Comput., 2015, 11, 2053-2062. 